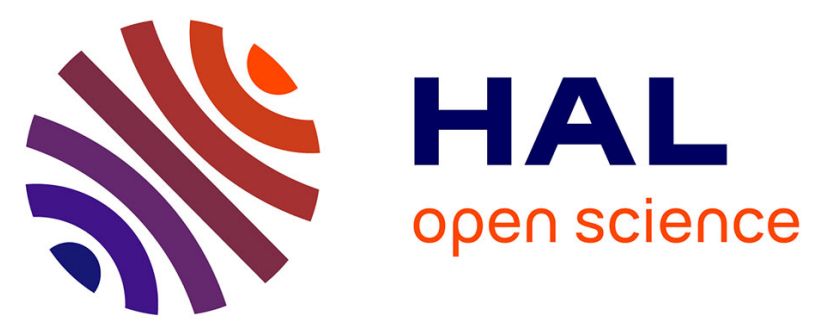

\title{
Magneto-optics of bilayer inclusions in multilayered epitaxial graphene on the carbon face of $\mathrm{SiC}$
}

Milan Orlita, Clement Faugeras, J. Borysiuk, J. M. Baranowski, W.

Strupinski, Mike Sprinkle, Claire Berger, Walt A. de Heer, D. M. Basko,

Gines Martinez Garcia, et al.

\section{To cite this version:}

Milan Orlita, Clement Faugeras, J. Borysiuk, J. M. Baranowski, W. Strupinski, et al.. Magneto-optics of bilayer inclusions in multilayered epitaxial graphene on the carbon face of SiC. Physical Review B: Condensed Matter and Materials Physics (1998-2015), 2011, 83 (12), pp.125302. 10.1103/PhysRevB.83.125302 . hal-01002909

\section{HAL Id: hal-01002909 https://hal.science/hal-01002909}

Submitted on 7 Jun 2014

HAL is a multi-disciplinary open access archive for the deposit and dissemination of scientific research documents, whether they are published or not. The documents may come from teaching and research institutions in France or abroad, or from public or private research centers.
L'archive ouverte pluridisciplinaire HAL, est destinée au dépôt et à la diffusion de documents scientifiques de niveau recherche, publiés ou non, émanant des établissements d'enseignement et de recherche français ou étrangers, des laboratoires publics ou privés. 


\title{
Magneto-optics of bilayer inclusions in multilayered epitaxial graphene on the carbon face of $\mathrm{SiC}$
}

\author{
M. Orlita, ${ }^{1,2, *}$ C. Faugeras, ${ }^{1}$ J. Borysiuk, ${ }^{3}$ J. M. Baranowski, ${ }^{4}$ W. Strupiński, ${ }^{5}$ M. Sprinkle, ${ }^{6}$ C. Berger, ${ }^{6,7}$ W. A. de Heer, ${ }^{6}$ \\ D. M. Basko, ${ }^{8}$ G. Martinez, ${ }^{1}$ and M. Potemski ${ }^{1}$ \\ ${ }^{1}$ Laboratoire National des Champs Magnétiques Intenses, CNRS-UJF-UPS-INSA, 25, avenue des Martyrs, F-38042 Grenoble, France \\ ${ }^{2}$ Institute of Physics, Faculty of Mathematics and Physics, Charles University, Ke Karlovu 5, 121 16 Praha 2, Czech Republic \\ ${ }^{3}$ Institute of Physics, Polish Academy of Sciences, 02-668 Warsaw, Al. Lotnikow 32/46, Poland \\ ${ }^{4}$ Institute of Experimental Physics, University of Warsaw, Hoża 69, PL 00-681 Warsaw, Poland \\ ${ }^{5}$ Institute of Electronic Materials Technology, PL 01-919 Warsaw, Poland \\ ${ }^{6}$ School of Physics, Georgia Institute of Technology, Atlanta, Georgia 30332, USA \\ ${ }^{7}$ Institut Néel/CNRS-UJF BP 166, F-38042 Grenoble Cedex 9, France \\ ${ }^{8}$ Laboratoire de Physique et Modélisation des Milieux Condensés, UJF and CNRS, F-38042 Grenoble, France
}

(Received 8 October 2010; published 14 March 2011)

\begin{abstract}
Additional component in multilayer epitaxial graphene grown on the C-terminated surface of SiC, which exhibits the characteristic electronic properties of a AB-stacked graphene bilayer, is identified in magnetooptical response of this material. We show that these inclusions represent a well-defined platform for accurate magnetospectroscopy of unperturbed graphene bilayers.
\end{abstract}

DOI: 10.1103/PhysRevB.83.125302

PACS number(s): 73.30.+y, 76.40.+b, 71.70.Di, 78.20.Ls

\section{INTRODUCTION}

The absence of the energetic gap in the excitation spectrum of graphene ${ }^{1}$ is considered as a possible drawback preventing the straightforward application of this emerging material in electronics. This is despite numerous efforts, such as those implying surface patterning ${ }^{2}$ and substrate- or adsorbentsinduced interactions. ${ }^{3-5}$ The possibility to open and tune the band gap in the bilayer graphene has recently been demonstrated by applying an electric field perpendicular to the graphitic planes ${ }^{6-8}$ and this is a key element to construct a transistor, the building block of electronic circuits. The band gap engineering is "typical" of the bilayer and is not reported in tri- and more-layer graphene specimens where semimetallic behavior dominates. ${ }^{9}$ From the viewpoint of applications, the bilayer graphene thus becomes almost equally appealing material as graphene itself.

Optical spectroscopy has played an important role in investigations of the bilayer graphene, ${ }^{6,10,11} \mathrm{as}$, for instance, it allows to directly visualize the electric-field induced energy gap in this system. ${ }^{7,8}$ On the other hand, only relatively scarce information has been up to now collected from magnetooptical measurements. ${ }^{12}$ This fact might be surprising when noticing the potential of Landau level (LL) spectroscopy which has been widely applied to other graphene-like systems. Magneto-optical methods have, for example, convincingly illustrated the unconventional LL spectrum in graphene, have offered a reliable estimate of the Fermi velocity or invoked the specific effects of many-body interactions between massless Dirac fermions. ${ }^{13-19}$

So far, the only magneto-optical experiments on the bilayer graphene have been reported by Henriksen et al., ${ }^{12}$ who succeeded to probe a relatively weak cyclotron-resonance signal of a small flake using the gate-controlled differential technique. The optical response at a fixed magnetic field was then studied as a function of the carrier density. Such differential spectroscopy was efficient in the case of exfoliated graphene monolayers, ${ }^{14,18}$ but it provides more complex results when applied to the bilayer graphene. In this latter system, the change of the gate voltage affects not only the carrier density but also modifies significantly the band structure and data interpretation is by far more elaborated. ${ }^{20}$

In this paper, we demonstrate that certain class of previously reported $\mathrm{AB}$-stacking faults ${ }^{21-24}$ in otherwise rotationallyordered multilayer epitaxial graphene (MEG), ${ }^{13,25-27}$ show the characteristic features of well-defined graphene bilayers. These inclusions, identified here in magnetotransmission experiments, represent therefore a suitable system for accurate magnetospectroscopy studies of unperturbed bilayer graphene.

\section{SAMPLE PREPARATION AND EXPERIMENTAL DETAILS}

The growth of MEG samples studied here was performed with a commercially available horizontal chemical vapor deposition hot-wall reactor (Epigress V508), inductively heated by a RF generator. Epitaxial MEG films were grown on semiinsulating $4 \mathrm{H}-\mathrm{SiC}(000 \overline{1})$ on-axis $\mathrm{C}$-terminated substrates at $1600^{\circ} \mathrm{C}$ in $\mathrm{Ar}$ atmosphere. The growth rate was controlled by the Ar pressure ( 100 mbar) which was found to directly influence the evaporation rate of $\mathrm{Si}$ atoms.

To measure the infrared transmittance of our samples, we used the radiation of a globar, which was analyzed by a Fourier transform spectrometer and delivered to the sample via lightpipe optics. The transmitted light was detected by a composite bolometer kept at $T=2 \mathrm{~K}$ and placed directly below the sample. Measurements were carried out in superconducting ( $B=0-13 \mathrm{~T})$ and resistive $(B=13-32 \mathrm{~T})$ solenoids with spectral resolution of 0.5 and $1 \mathrm{meV}$ in the range of magnetic field below and above $B=13 \mathrm{~T}$, respectively. All presented spectra were normalized by the sample transmission at $B=0$.

The samples were characterized in micro-Raman scattering experiments which, similarly to previous studies, ${ }^{21}$ revealed, depending on location, single-component 2D band features, characteristic of graphene simple electronic bands and of decoupled graphitic planes in multilayer epitaxial graphene grown on the $\mathrm{C}$-face of a $\mathrm{SiC}$ substrate, or multicomponent 
2D band features characteristic of Bernal stacked graphite. In this paper, we present transmission data obtained on one particular specimen with a high number of graphitic layers $(\sim 100)$ and grown on a SiC substrate with a reduced thickness of $\sim 100 \mu \mathrm{m}$. Due to this latter condition, the spectral region of total opacity of the sample only covers the $\mathrm{SiC}$ reststrahlen band ( $~ 85-120 \mathrm{meV})$, i.e., it is significantly narrower as compared to the case of the preceding studies. ${ }^{28,29}$ In spite of these efforts to expand the available spectral range, a relatively weak transmission was still found around the energy of $200 \mathrm{meV}$, due to double-phonon absorptions in the underlying $\mathrm{SiC}$ substrate and transmission spectra are affected by strong interference patterns due to the relatively thin substrate. These two effects prevented measurements in the energy range below the reststrahlen band of $\mathrm{SiC}$.

\section{RESULTS AND DISCUSSION}

Typical transmission spectra of the investigated sample are shown in Fig. 1. The dominant absorption lines which are observed in these spectra show the characteristic $\sqrt{B}$ - dependence (see Fig. 2) and correspond to inter-LL transitions in electrically isolated graphene sheets. We denote those lines by Roman letters, following the initial work and notation of Sadowski et al. ${ }^{13,28}$ These dominant spectral features are equivalent to the characteristic lines observed in the magnetooptical response of exfoliated graphene monolayers. ${ }^{14,15,18}$ The subsequent absorption lines labeled here as $B \rightarrow I$ correspond to transitions $L_{-m(-m-1)} \rightarrow L_{m+1(m)}$ with $m=$ $0 \rightarrow 7$ between LLs in graphene: $E_{m}=\operatorname{sign}(m) E_{1} \sqrt{|m|}$, where $E_{1}=v_{F} \sqrt{2 \hbar|e B|}$. The apparent Fermi velocity is extracted to be $v_{F}=(1.02 \pm 0.02) \times 10^{6} \mathrm{~m} / \mathrm{s}$. Intriguingly, the $L_{-1(0)} \rightarrow L_{0(1)}$ transition exhibits a significant broadening above $16 \mathrm{~T}$, which could be tentatively related to electronphonon interaction. This effect will be discussed elsewhere.

The main focus of the present work are other spectral features, i.e., the transmission dips denoted by $\mathbf{n}=\mathbf{1}, \mathbf{n}=\mathbf{2}, \ldots, \mathbf{n}=\mathbf{5}$ in Fig. 1. These absorption lines are significantly weaker than the dominant "graphene lines," but are still well resolved in our spectra. As it can be seen in Fig. 2, in contrast to the dominant transitions, these weaker lines follow a nearly linear in $B$ dependence. As this behavior is characteristic of massive particles and because graphene bilayer is the simplest graphene based system with such particles, we anticipate that electronic excitations within graphene bilayer inclusions are responsible for the $\mathbf{n}=\mathbf{1}, \mathbf{n}=\mathbf{2}, \ldots, \mathbf{n}=\mathbf{5}$ transitions. The energy ladder $\varepsilon_{n, \mu}$ of LLs in a graphene bilayer can be easily calculated ${ }^{30,31}$ within the standard four band model which only considers the two most relevant coupling constants $\gamma_{0}$ and $\gamma_{1}$ (see, e.g., Ref. 32 for their definitions):

$$
\begin{aligned}
\varepsilon_{n, \mu}= & \operatorname{sign}(n) \frac{1}{\sqrt{2}}\left[\gamma_{1}^{2}+(2|n|+1) E_{1}^{2}\right. \\
& \left.+\mu \sqrt{\gamma_{1}^{4}+2(2|n|+1) E_{1}^{2} \gamma_{1}^{2}+E_{1}^{4}}\right]^{1 / 2} .
\end{aligned}
$$

Here, a positive (negative) integer $n$ indexes the electron (hole) LLs. $\mu=-1$ accounts for the topmost-valence and the lowest-conduction band, whereas $\mu=1$ corresponds to two other, split-off bands. As illustrated in the inset of Fig. 2, optically active inter Landau level transitions in a graphene bilayer fulfill the $|n| \rightarrow|n| \pm 1$ selection rule. The energies of such transitions are plotted in Fig. 2 with black solid lines. Those lines account for the transitions within the $\mu=-1$ bands. To reproduce the experimental data, we have adjusted the $\gamma_{1}$ parameter whereas the Fermi velocity $v_{F}$ which defines $E_{1}$ (i.e., the intralayer coupling $\gamma_{0}=3150 \mathrm{meV}$ ) has been fixed at the value derived from the monolayer-like transitions. A fair agreement is obtained between the calculated (solid lines in Fig. 2) and measured energies of $\mathbf{n}=\mathbf{1}, \mathbf{n}=\mathbf{2}, \ldots, \mathbf{n}=\mathbf{5}$ transitions. Optical absorptions involving LLs of higher indexes (e.g., $\mathbf{n}=\mathbf{6}, \mathbf{n}=\mathbf{7}$, and $\mathbf{n}=\mathbf{8}$, see Fig. 2) could also be observed in the spectra, nevertheless, these lines are very

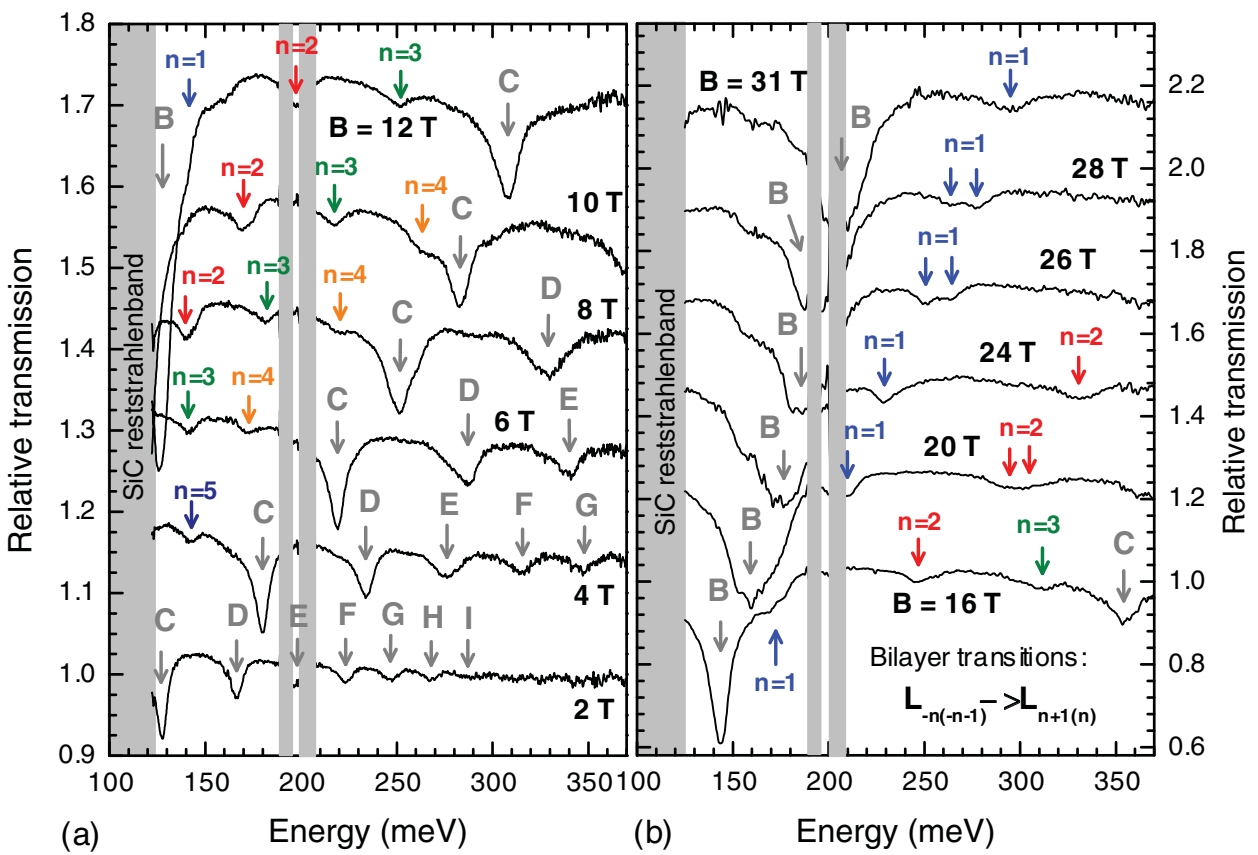

125302-2 (a) Energy $(\mathrm{meV})$

(b)
Energy (meV)
FIG. 1. (Color online) Transmission spectra of MEG with $\sim 100$ layers recorded at the selected magnetic fields below 12 and above $16 \mathrm{~T}$ in (a) and (b), respectively, in both cases above the reststrahlen band of $\mathrm{SiC}$. Whereas the transitions marked $\mathrm{B}$ to I correspond to electrically isolated graphene monolayers, transitions denoted $n=1$ to 5 match to interLL transitions in an unperturbed graphene bilayer, following the coding $L_{-n(-n-1)} \rightarrow L_{n+1(n)}$. For clarity, successive spectra in parts (a) and (b) are shifted vertically by 0.14 and 0.23 , respectively. 


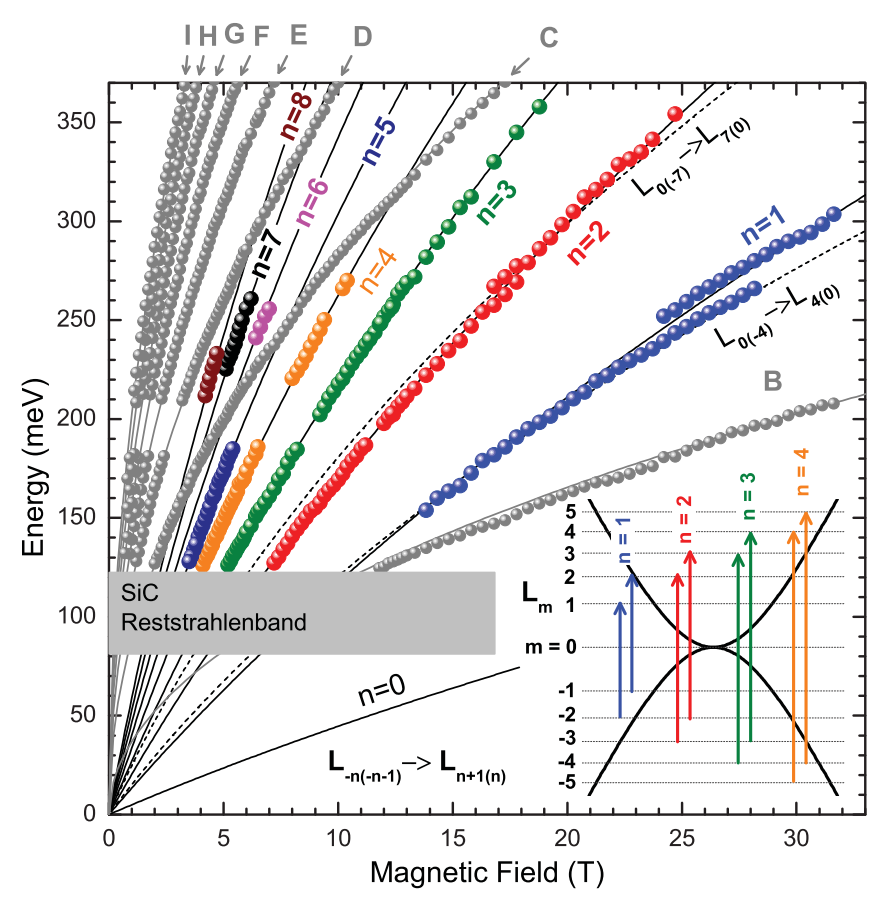

FIG. 2. (Color online) Fan chart: Points marked with Roman letters, having a characteristic $\sqrt{B}$ dependence, correspond to inter-LL transitions in electrically isolated graphene sheets. ${ }^{13}$ Points denoted by index $n$ represent interband inter-LL transitions in the graphene bilayer $L_{-n(-n-1)} \rightarrow L_{n+1(n)}$, as schematically shown in the inset. The full gray lines show expected energies of transitions in the graphene monolayer for $v_{F}=1.02 \times 10^{6} \mathrm{~m} / \mathrm{s}$, full black lines correspond to predicted positions of the absorption lines in the graphene bilayer (only parameters $\gamma_{0}=3150 \mathrm{meV}$ and $\gamma_{1}=$ $385 \mathrm{meV}$ considered). The dashed lines denote theoretical positions of two trigonal-warping-induced transitions in the graphene bilayer $L_{0(-4)} \rightarrow L_{4(0)}$ and $L_{0(-7)} \rightarrow L_{7(0)}$.

weak and only visible in a limited range of magnetic fields. Traces of inter-LL transitions due to split-off bands $[\mu=1$ in Eq. (1)], can be also identified in our data and experiments focused on this particular set of transitions are in progress.

A pronounced departure of the observed bilayer transitions from the linearity in $B$ clearly shows the limits of the parabolic approximation which is often used for graphene bilayers in the close vicinity of the $\mathbf{k}=0$ point, and within which the LLs are strictly linear with the magnetic field. ${ }^{32,33}$ As can be seen in Fig. 2, the positions of all these lines can be very well reproduced with a parameter $\gamma_{1}=(385 \pm 5) \mathrm{meV}$, and these experiments thus refine the value of this parameter reported previously from optical studies at zero magnetic field. ${ }^{6,10,11}$ The intriguing splitting of the $\mathbf{n}=\mathbf{1}$ and of the $\mathbf{n}=\mathbf{2}$ lines at high magnetic fields is beyond our simple model and will be discussed later on.

The simplified model of LLs in the pristine bilayer graphene provides us with reasonably accurate description of its magneto-optical response, even though it neglects the electron-hole asymmetry (mainly induced by tight-binding $\gamma_{4}, \Delta^{\prime}$ parameters), ${ }^{6,8,10,11}$ as well as a possible gap opening at the charge neutrality point. Nevertheless, it should be noted that the optical response of the graphene bilayer has only been unambiguously identified above the reststrahlen band of the $\mathrm{SiC}$ substrate and therefore, we cannot exclude a possible appearance of an energy gap, up to a few tens of meV, at the $\mathbf{k}=0$ point. For the same reason, we can only estimate a very higher limit for the carrier density in the studied bilayer of $2 \times 10^{12} \mathrm{~cm}^{-2}$. However, the real carrier density is very likely similar to that of the surrounding (electrically isolated) graphene sheets, i.e., below $10^{10} \mathrm{~cm}^{-2}$, as reported in Refs. 16 and 22. We also point out that relatively narrow line widths of the order of $10 \mathrm{meV}$ (relaxation time in sub-picosecond range) serve as an indication of rather high electronic quality of these bilayer inclusions, comparable or even better than other bilayer systems. ${ }^{12,34,35}$

Equivalent bilayer-like spectral features are recurrently identified practically in all studied specimens, nevertheless, with a strongly varying intensity. In general, we can say that the relative intensity of these "bilayer" lines increases with the total number of layers in MEG and these transitions are practically invisible in specimens with less than ten layers reported in the very first magnetospectroscopy studies. ${ }^{13,28}$ This finding serves as an indication that we indeed observe graphene bilayer inclusions and not regions of a local $\mathrm{AB}$ stacking which might be also speculated to appear in rotationally stacked multilayers. Such Moiré-patterned AB-stacked areas have been recently visualized in MEG by STM/STS measurements. ${ }^{36-38}$ We further assume that twisted graphene layers which results in the Moiré patterned bilayer should not provide us with so well-defined $A B$ stacked bilayers as we observe in our data. Let us also note that if we compare the relative intensity of observed transitions, we can roughly estimate that in none of the investigated samples the ratio between bilayers and monolayers exceeded $10 \%$.

We should also emphasize that the appearance of Bernalstacked faults in MEG, which have a form of well-defined bilayers, is not a signature of bulk graphite. In this well-known material, the $K$-point electrons indeed mimic massive carriers in the graphene bilayer, but with an effective interlayer coupling $2 \gamma_{1}$ instead of $\gamma_{1}$ in a real graphene bilayer. ${ }^{31,39-41}$ This twofold coupling in the effective bilayer model for $K$-point electrons implies a characteristic effective mass twice enhanced in comparison to that of massive Dirac fermions in true graphene bilayer and consequently, also a twice lower energy separation between adjacent interband inter-LL transitions, cf. Fig. 2 of this paper with the fan chart in Ref. 40.

The remaining unclarified point of our study is the splitting of the bilayer lines, which is clearly visible for transitions $\mathbf{n}=\mathbf{1}$ and $\mathbf{n}=\mathbf{2}$ around $B=17$ and $26 \mathrm{~T}$, respectively. In the following, we discuss two different scenarios for this splitting. One possible explanation invokes the electron-hole asymmetry, reported recently in graphene bilayers graphene. $6,10,11$ Based on this assumption, the magnitude of the splitting for the $n$th transition, relative to the transition energy is expressed by ${ }^{42}$

$$
\frac{2\left(\Delta^{\prime} / \gamma_{1}+2 \gamma_{4} / \gamma_{0}\right)}{\sqrt{n(n+1)}+\sqrt{(n+1)(n+2)}} .
$$

For the values $\Delta^{\prime}=0.02 \mathrm{eV}, \quad \gamma_{1}=0.4 \mathrm{eV}, \quad \gamma_{4} / \gamma_{0}=$ $0.05,{ }^{6-8,10,11}$ our measured value for $n=1$ (about 0.08 ) is very well reproduced. However, the splitting due to electron-hole asymmetry should be seen for all magnetic fields, while, as can 
be seen in Fig. 1b, we only observe it in a relatively narrow range of $B$.

Perhaps a more natural explanation for this line splitting would be an avoided crossing between the transition $L_{-n(-n-1)} \rightarrow L_{n+1(n)}$ and some other transition with a much smaller oscillator strength, so that it is not seen far from the crossing point. One can see directly from Fig. 2 that the bright transitions $n=1,2$ are crossed by the dark (i.e., dipole-inactive in case of a zero trigonal warping) transitions $L_{0(-4)} \rightarrow L_{4(0)}, L_{0(-7)} \rightarrow L_{7(0)}$, respectively, approximately at the observed values of $B$ (the crossing occurs at a very sharp angle, which brings a significant uncertainty). These transitions are allowed only due to the presence of the trigonal warping of the electronic bands, which mixes levels $\mathrm{L}_{m}$ with $|m|$ differing by an integer multiple of 3 , see Ref. 30 . The ratio of the oscillator strength of the $L_{0(-4)} \rightarrow L_{4(0)}$ transition to that of the bright $n=1$ transition can be estimated ${ }^{42}$ as $(25 / 108)\left(\gamma_{3} \gamma_{1} / \gamma_{0}\right)^{2}\left(l_{B} / \hbar v_{F}\right)^{2} \simeq 0.02$ at $B=25 \mathrm{~T}$. The $L_{0(-4)} \rightarrow L_{4(0)}$ transition is therefore not expected to be seen in the experiment unless some other, possibly resonant, admixture mechanism is taken into account. Coupling between $L_{0(-4)} \rightarrow L_{4(0)}$ and $n=1$ transitions should be quite strong as the observed "anticrossing splitting" is of about $20 \mathrm{meV}$.

We have speculated this mode coupling could be due to electron-phonon or electron-electron interactions. Electronphonon interaction, which could be enhanced due to the proximity of the transition energy $(250 \mathrm{meV})$ to that of the zone-center optical phonon (196 meV), must be excluded due to the different symmetry (this phonon is Raman active). Splitting due to Coulomb interaction can be evaluated to be $0.04\left(e^{2} / 4 \pi \varepsilon_{0} \hbar v_{F}\right)\left(\gamma_{1} \gamma_{3} / \gamma_{0}\right),{ }^{42}$ i.e., only about $3 \mathrm{meV}$ in the absence of dielectric screening, $e^{2} / 4 \pi \varepsilon_{0} \hbar v_{F}=2.2$. Hence, the mechanism of the possible strong coupling between the $L_{-1(-2)} \rightarrow L_{2(1)}$ and $L_{0(-4)} \rightarrow L_{4(0)}$ transitions is a puzzle which remains to be clarified.

\section{CONCLUSIONS}

We probed graphene bilayers embedded in multilayer epitaxial graphene on the $\mathrm{C}$-terminated surface of SiC. These inclusions can be viewed as AB-stacked faults in an otherwise rotationally stacked multilayer graphene structure and enable spectroscopic studies of unperturbed graphene bilayers. The "electronic quality" of these bilayers is comparable or even better than that of the bilayers obtained by exfoliation or by epitaxial growth on the Si-terminated surface of SiC. ${ }^{34,35}$ This way, we could trace the interband inter-LL transitions in the graphene bilayer for the first time, and thus supply data complementary to the cyclotron resonance absorption (i.e., intraband inter-LL transitions) measured on the exfoliated bilayer by Henriksen et al. ${ }^{12}$ We could also clearly visualize the departure of Landau levels in the graphene bilayer from the linearity in $B$, which clearly sets limits for the parabolic approximation of electronic bands in this material.

\section{ACKNOWLEDGMENTS}

We acknowledge funding received from EC-EuroMagNetII under Contract No. 228043, from the Keck Foundation, and from the Partner University Fund at the Embassy of France. This work has been supported by the Projects No. 395/NPICSFR/2009/0, MSM0021620834, GACR Nos. P204/10/1020 and MTKD-CT-2005-029671, furthermore by Grants No. 670/NESF-EPI/2010/0, 671/NESF-EPI/2010/0, and GRA/10/E006 within the ESF EuroGraphene programme (EPIGRAT). *milan.orlita@1ncmi.cnrs.fr; also at Institute of Physics, v.v.i., ASCR, Prague, Czech Republic.

${ }^{1}$ K. S. Novoselov, Nature Mater. 6, 720 (2007).

${ }^{2}$ J. Bai, X. Zhong, S. Jiang, Y. Huang, and X. Duan, Nature Nanotechnology 5, 190 (2010).

${ }^{3}$ S. Y. Zhou, G.-H. Gweon, A. V. Fedorov, P. N. First, W. A. de Heer, D.-H. Lee, F. Guinea, A. H. C. Neto, and A. Lanzara, Nature Mater. 6, 770 (2007).

${ }^{4}$ A. Bostwick, T. Ohta, T. Seyller, K. Horn, and E. Rotenberg, Nature Phys. 3, 36 (2007).

${ }^{5}$ R. Balog, B. Jorgensen, L. Nilsson, M. Andersen, E. Rienks, M. Bianchi, M. Fanetti, E. Lagsgaard, A. Baraldi, S. Lizzit et al., Nat. Mater. 9, 315 (2010).

${ }^{6}$ A. B. Kuzmenko, E. van Heumen, D. van der Marel, P. Lerch, P. Blake, K. S. Novoselov, and A. K. Geim, Phys. Rev. B 79, 115441 (2009).

${ }^{7}$ K. F. Mak, C. H. Lui, J. Shan, and T. F. Heinz, Phys. Rev. Lett. 102, 256405 (2009).

${ }^{8}$ Y. Zhang, T.-T. Tang, C. Girit, Z. Hao, M. C. Martin, A. Zettl, M. F. Crommie, Y. R. Shen, and F. Wang, Nature (London) 459, 820 (2009).

${ }^{9}$ M. F. Craciun, S. Russo, M. Yamamoto, J. B. Oostinga, A. F. Morpurgo, and S. Tarucha, Nature Nanotech. 4, 383 (2009).
${ }^{10}$ L. M. Zhang, Z. Q. Li, D. N. Basov, M. M. Fogler, Z. Hao, and M. C. Martin, Phys. Rev. B 78, 235408 (2008).

${ }^{11}$ Z. Q. Li, E. A. Henriksen, Z. Jiang, Z. Hao, M. C. Martin, P. Kim, H. L. Stormer, and D. N. Basov, Phys. Rev. Lett. 102, 037403 (2009).

${ }^{12}$ E. A. Henriksen, Z. Jiang, L.-C. Tung, M. E. Schwartz, M. Takita, Y.-J. Wang, P. Kim, and H. L. Stormer, Phys. Rev. Lett. 100, 087403 (2008).

${ }^{13}$ M. L. Sadowski, G. Martinez, M. Potemski, C. Berger, and W. A. de Heer, Phys. Rev. Lett. 97, 266405 (2006).

${ }^{14}$ Z. Jiang, E. A. Henriksen, L. C. Tung, Y.-J. Wang, M. E. Schwartz, M. Y. Han, P. Kim, and H. L. Stormer, Phys. Rev. Lett. 98, 197403 (2007).

${ }^{15}$ R. S. Deacon, K.-C. Chuang, R. J. Nicholas, K. S. Novoselov, and A. K. Geim, Phys. Rev. B 76, 081406(R) (2007).

${ }^{16}$ M. Orlita, C. Faugeras, P. Plochocka, P. Neugebauer, G. Martinez, D. K. Maude, A.-L. Barra, M. Sprinkle, C. Berger, W. A. de Heer et al., Phys. Rev. Lett. 101, 267601 (2008).

${ }^{17}$ P. Neugebauer, M. Orlita, C. Faugeras, A.-L. Barra, and M. Potemski, Phys. Rev. Lett. 103, 136403 (2009).

${ }^{18}$ E. A. Henriksen, P. Cadden Zimansky, Z. Jiang, Z. Q. Li, L.-C. Tung, M. E. Schwartz, M. Takita, Y.-J. Wang, P. Kim, and H. L. Stormer, Phys. Rev. Lett. 104, 067404 (2010). 
${ }^{19}$ I. Crassee, J. Levallois, A. L. Walter, M. Ostler, A. Bostwick, E. Rotenberg, T. Seyller, D. van der Marel, and A. B. Kuzmenko, Nature Physics 7, 48 (2011).

${ }^{20}$ M. Mucha-Kruczynski, E. McCann, and V. Fal'ko, Solid State Commun. 149, 1111 (2009).

${ }^{21}$ C. Faugeras, A. Nerrière, M. Potemski, A. Mahmood, E. Dujardin, C. Berger, and W. A. de Heer, Appl. Phys. Lett. 92, 011914 (2008).

${ }^{22}$ M. Sprinkle, D. Siegel, Y. Hu, J. Hicks, A. Tejeda, A. Taleb Ibrahimi, P. Le. Fèvre, F. Bertran, S. Vizzini, H. Enriquez et al., Phys. Rev. Lett. 103, 226803 (2009).

${ }^{23}$ M. Sprinkle, J. Hicks, A. Tejeda, A. Taleb-Ibrahimi, P. L. Fèvre, F. Bertran, H. Tinkey, M. Clark, P. Soukiassian, D. Martinotti, J. Hass, and E. H. Conrad J. Phys. D: Appl. Phys. 43, 374006 (2010).

${ }^{24}$ D. A. Siegel, C. G. Hwang, A. V. Fedorov, and A. Lanzara, Phys. Rev. B 81, 241417 (2010).

${ }^{25}$ C. Berger, Z. Song, T. Li, X. Li, A. Y. Ogbazghi, R. Feng, Z. Dai, A. N. Marchenkov, E. H. Conrad, P. N. First et al., J. Phys. Chem. B 108, 19912 (2004).

${ }^{26}$ J. Hass, F. Varchon, J. E. Millán-Otoya, M. Sprinkle, N. Sharma, W. A. de Heer, C. Berger, P. N. First, L. Magaud, and E. H. Conrad, Phys. Rev. Lett. 100, 125504 (2008).

${ }^{27}$ D. L. Miller, K. D. Kubista, G. M. Rutter, M. Ruan, W. A. de Heer, P. N. First, and J. A. Stroscio, Science 324, 924 (2009).

${ }^{28}$ M. L. Sadowski, G. Martinez, M. Potemski, C. Berger, and W. A. de Heer, Solid State Commun. 143, 123 (2007).
${ }^{29}$ P. Plochocka, C. Faugeras, M. Orlita, M. L. Sadowski, G. Martinez, M. Potemski, M. O. Goerbig, J.-N. Fuchs, C. Berger, and W. A. de Heer, Phys. Rev. Lett. 100, 087401 (2008).

${ }^{30}$ D. S. L. Abergel and V. I. Fal'ko, Phys. Rev. B 75, 155430 (2007).

${ }^{31}$ M. Koshino and T. Ando, Phys. Rev. B 77, 115313 (2008).

${ }^{32}$ E. McCann and V. I. Fal'ko, Phys. Rev. Lett. 96, 086805 (2006).

${ }^{33}$ K. S. Novoselov, E. McCann, S. V. Morozov, V. I. Fal'ko, K. I. Katsnelson, U.Zeitler, D. Jiang, F. Schedin, and A. K. Geim, Nature Phys. 2, 177 (2006).

${ }^{34}$ T. Ohta, A. Bostwick, T. Seyller, K. Horn, and E. Rotenberg, Science 313, 951 (2006).

${ }^{35}$ C. Riedl, C. Coletti, T. Iwasaki, A. A. Zakharov, and U. Starke, Phys. Rev. Lett. 103, 246804 (2009).

${ }^{36}$ D. L. Miller, K. D. Kubista, G. M. Rutter, M. Ruan, W. A. de Heer, P. N. First, and J. A. Stroscio, Phys. Rev. B 81, 125427 (2010).

${ }^{37}$ D. L. Miller, K. D. Kubista, G. M. Rutter, M. Ruan, W. A. de Heer, M. Kindermann, P. N. First, and J. A. Stroscio, Nature Physics 6, $811(2010)$

${ }^{38}$ M. Kindermann and P. N. First, Phys. Rev. B 83, 045425 (2011).

${ }^{39}$ B. Partoens and F. M. Peeters, Phys. Rev. B 75, 193402 (2007).

${ }^{40}$ M. Orlita, C. Faugeras, J. M. Schneider, G. Martinez, D. K. Maude, and M. Potemski, Phys. Rev. Lett. 102, 166401 (2009).

${ }^{41}$ K.-C. Chuang, A. M. R. Baker, and R. J. Nicholas, Phys. Rev. B 80, 161410(R) (2009).

${ }^{42}$ This expression is obtained in the two-band approximation. The degree of error introduced by this approximation at energies about $250 \mathrm{meV}$ can be roughly estimated from the deviation of the curves in Fig. 2 from their low-field tangents, which gives about $30 \%$. 